\section{MANAGING PERISHABLE INVENTORY}

\author{
OPHER BARON \\ Rotman School of Management, \\ University of Toronto, Toronto,
} Ontario, Canada

\section{INTRODUCTION}

This article discusses inventory management of perishable items, including the differences between inventory models that consider items' perishability and the standard models that ignore this issue. We hope that this article will help fostering the interest of students and researchers in inventory control of perishable items, and ultimately help improving the inventory control of perishable items in practice.

To emphasize the importance of appropriately managing inventory of perishables, note that Ref. 1 estimates the total cost of unsalable merchandise of suppliers to drugstores and supermarkets in 2005 by $\$ 2.05$ billion. This cost goes directly to the bottom line of many of these companies. Moreover, higher clock speed in many industries results in shorter and shorter life-cycles of many products. The inventory control of such products might become similar to the one of perishable items as well after some period, when a newer version is presented, the value of old items declines significantly. One important difference between items with short life-cycle and perishables is that in some cases, the firm controls the introduction of the new technology. Still, much of the insight derived for managing perishable inventory may be valid for products with short life cycles.

Most of the traditional inventory models analyzed in the literature (e.g., $\mathrm{EOQ},(Q, r)$, and $(S, s)$ policies) assume an infinite shelflife for items (see Multiechelon Multiproduct Inventory Management). (An exception is the newsvendor model discussed in the section titled "Extending the Newsvendor Model".) Thus, when the shelf-life of the items stored is finite, traditional control policies may not be sufficient. For this reason, special purpose models that focus on the management of inventory of perishable items were developed. Our purpose is to highlight the reasons for these differences and enable further investigation of the models developed for perishable items by providing relevant references. A more technical discussion of the techniques employed in the analysis of perishable items is given in the article titled Mathematical Models for Perishable Inventory Control in this encyclopedia.

This article continues as follows: the section titled "Modeling Inventory Problems for Perishable Items" highlights the differences between inventory models for perishables and models that ignore perishability. The section titled "Taxonomy of Inventory Models for Perishable Items with Infinite Horizon" provides a taxonomy of models for controlling inventory of perishables based on Ref. 2, and lists some of the basic results of such models. An important observation from this section is that effective heuristics are required for the management of perishable items. Thus, the section titled "Developing a Heuristic Control Policy" demonstrates the development of such a heuristic based on Ref. 3, and finally, the section titled "Future Research Directions" lists probable directions for future research on the management of perishable items.

\section{MODELING INVENTORY PROBLEMS FOR PERISHABLE ITEMS}

The analysis of inventory systems is primarily focused on the tactical question of which inventory control policies to use and the operational questions of when and how much inventory to order. By and large, these are the main questions for managing the inventory of perishable items as well. This section reminds the reader of the assumptions used

Wiley Encyclopedia of Operations Research and Management Science, edited by James J. Cochran

Copyright $\odot 2010$ John Wiley \& Sons, Inc. 
in modeling standard inventory systems for nonperished items and then lists the special features of models for perishables. It concludes by generalizing the newsvendor model to consider items with finite shelf-life. In the process, we present relevant notation.

\section{Inventory Modeling for Nonperishables}

In inventory models for nonperishables, time is measured either in a discrete (i.e., periodic) or continuous fashion. The choice of time measurement is often related to the review period (periodic or continuous) and to the model horizon, $T$, that may be a single period, finite, or infinite horizon. The replenishment lead time, $L$, may be assumed to be zero, deterministic, or stochastic (with or without order crossings). The customers arrival rate per time period, $\lambda$, may be deterministic or stochastic, each individual's demand, $D$, may be deterministic or stochastic, and $D$ may be discrete or continuous. A typical assumption is that the arrival process is Poisson with rate $\lambda ; D=1$ (see Poisson Process and its Generalizations). Finally, when there are no items on the shelf, that is there are shortages, demand is typically assumed to be backlogged or lost.

The costs considered in the management of standard inventory systems are per unit procurement cost, $c$; per unit selling price, $p$; order setup cost, $K \geq 0$; and holding cost per unit per time period, $h>0$. Another cost is that of shortages. The shortage costs can include a one-time cost per shortage, $K_{b} \geq 0$ if shortages are backlogged, or $K_{l} \geq 0$ when shortages result in lost sales, and a cost per unit per unit time, $c_{b} \geq 0$, in cases of backlog, or a cost per unit, $c_{l} \geq 0$, in the case of lost sales.

\section{Special Features of Models of Perishables}

In addition to the above list of characteristics, modeling of inventory for perishable items also requires a characterization of the time to perishability, $L_{p}$. This time to perishability may be either deterministic or stochastic. Moreover, when orders arrive in batches, all items in a batch may share the same time to perishability, or each item may have its own shelf-life. An alternative assumption is that the inventory of good items is deteriorating with time at some rate, as in Ref. 4.

The two most common models for perishability are outdatedness due to reaching expiry date (e.g., food items or medicine) and sudden perishability due to disaster (e.g., spoilage because of extreme weather conditions). The perishability due to outdatedness is typically modeled as a deterministic time to perishability and the perishability due to disaster is typically modeled as an exponential (or its discrete counterpart, geometric) time to perishability. This is because the memory-less property of these distributions often results in more tractable models.

The treatment of lead time in the management of inventory for perishable items is also not trivial. One difficulty is that the items might perish during the delivery time. This might be addressed by assuming that the supplier supplies fresh items upon their delivery and changes their lead time accordingly. For example, when the lead time is deterministic and items' shelf-life is exponential, we can assume that delivered items are fresh by modeling the lead time as a geometric random variable.

In the example above, changing the lead time to support the assumption that supplied items are fresh complicates the model because it introduces another uncertainty in the model; namely, the corrected lead time becomes uncertain. To avoid complicating the model, much of the literature on perishable items assumes that supplied items (after the lead time) are fresh.

Another complication is that when lead times are long relative to the items' shelflives, there might be several orders out at the same time. Then, orders might cross each other. This potentially affects the shelflife distribution of delivered items. (Order crossing typically increases the complexity of traditional models as well.)

A general difficulty caused by the presence of lead time in a stochastic environment is that shelf-life of items in stock depends on which batch they arrived at. Thus, the controller should keep track not only of the quantity of the items on shelf but also the length of time different items are on the shelf; that is, their age and its distribution. 
Another challenge in the analysis of perishable items is that items that have not yet perished may have different remaining shelf-life. (Either because items have a unique shelf-life, or because batches with common shelf-lives arrived in different periods.) Thus, the information required to completely characterize the on-hand and on-order inventory includes not only the quantity of inventory but also the remaining shelf-lives of each unit in the inventory. This increase in information requirement makes the analysis of multiechelon supply chains for perishable products especially challenging (because even for standard items, such an analysis often relies on dynamic programming and suffers from the curse of dimensionality). Therefore, some important theoretical contributions developed in the analysis of perishable items address the information required to characterize the inventory level process. (See the discussion in the section titled "Models Without Order Setup Cost or Lead Times" and in the article titled Mathematical Models for Perishable Inventory Control in this encyclopedia).

Another relevant decision in managing perishables when items of several different ages coexist, is items dispatching to fulfill orders. In such cases, it might not be optimal to dispatch products in a first-in-first-out fashion. For an extreme case, consider the following example.

Example 1. A last-in-first-out dispatching policy would decrease the effect of perishability when an item's shelf-life follows a new worse-than-used distribution. (Let $F_{L_{p}}(s)$ denote the cumulative distribution function of the shelf-life. Then, if

$$
\begin{array}{r}
1-F_{L_{p}}(s+t) \geq\left(1-F_{L_{p}}(s)\right)\left(1-F_{L_{p}}(t)\right) \\
\forall s \geq 0, \forall t \geq 0,
\end{array}
$$

we say that $L_{p}$ has a new worse-than-used distribution.)

While perishable items with a new worsethan-used shelf-life are hard to find in practice, this example highlights the difficulty of characterizing the optimal dispatching policy. To substantially complicate the exact analysis of the optimal control policy for perishables, it is enough that a newly arriving batch may have a shorter shelf-life than that of existing items. Moreover, in cases when customers may control the items' dispatching they may prefer fresher items (consider items such as milk), causing the actual dispatching to differ from first-in-first-out.

\section{Extending the Newsvendor Model}

The newsvendor model discussed in the article titled Newsvendor Models in this encyclopedia is an exception in the standard inventory literature, because it considers perishability. This model considers the order quantity required to maximize the profit over a single period when the demand over the period is uncertain. In addition to the costs listed above, the newsvendor model also considers the salvage cost of perishable items $c_{s}$, which can be negative or positive.

The newsvendor model can also be used to model perishable items that can be ordered only once. Now, we model the length of the single period (i.e., the lifetime) as uncertain, but there are no further procurement opportunities. In this case, the uncertainty in the demand depends on both, the uncertain demand per time period and the uncertain length of the selling season. A similar idea was first investigated in Ref. 5, which in contrast to the newsvendor model, allows several ordering opportunities before the perishability time.

To express the uncertainty in demand in these settings, we assume that the shelflife $L_{p}$ is a discrete random variable with a $z$-transform $Z_{L_{p}}(z)=\sum_{i=0}^{\infty} z^{i} \operatorname{Pr}\left(L_{p}=i\right)$ and that the demand in time period $i$ is independent and identically distributed, $D_{i}$, with a probability density function (pdf), $f_{D_{i}}(x)$, and a moment-generating function, $L_{D_{i}}^{*}(\alpha)=$ $\int_{-\infty}^{\infty} \mathrm{e}^{\alpha x} f_{D_{i}}(x) \mathrm{d} x$. Then, the horizon considered by the planner is $T=L_{p}$, the overall demand during the period, $D_{T}$, is a random sum of random variables with a momentgenerating function $L_{D_{T}}^{*}$ :

$$
L_{D_{T}}^{*}(\alpha)=E\left(L_{D_{i}}^{*}(\alpha)^{L_{p}}\right)=Z_{L_{p}}\left(L_{D_{i}}^{*}(\alpha)\right)
$$


Example 2. If $L_{p} \sim \operatorname{Poisson}(p)$ and $D_{i}$ $\sim \operatorname{Normal}\left(\mu, \sigma^{2}\right)$ and are i.i.d, Then $Z_{L_{p}}(z)=e^{p(z-1)}, L_{D_{i}}^{*}(\alpha)=e^{\left(\mu \alpha+(\sigma \alpha)^{2} / 2\right)}$ and the moment-generating function of overall demand over the period is

$$
L_{D_{T}}^{*}(\alpha)=e^{p\left(\exp \left(\mu \alpha+(\sigma \alpha)^{2} / 2\right)-1\right)}
$$

Because the moment-generating function fully characterizes the demand distribution over the products' random shelf-life, the standard solution of the newsvendor model can be implemented to maximize the profit even when the valuable shelf-life of the items is uncertain.

\section{TAXONOMY OF INVENTORY MODELS FOR PERISHABLE ITEMS WITH INFINITE HORIZON}

As shown above, the newsvendor model is useful in the inventory management of perishable items that can only be ordered once. However, many perishable items such as food and medicines are consumed over a much longer horizon; then a single order is not practical. Finite horizon models were addressed in the literature almost solely in the discrete review settings. In contrast, infinite horizon models were considered in both discrete and continuous review settings. Below, we describe the primary models, assumptions, and results in the inventory literature on perishable items. We follow the classification of Ref. 2 in the section titled "Taxonomy of Inventory Models for Perishable Items with Infinite Horizon" because it is both thorough and recent. The interested reader is encouraged to read Ref. 2 .

The infinite horizon models for perishables include a cost, $c_{p}$, per perished item. This cost replaces the salvage value of perished items in the newsvendor model. It is also possible to include a fixed cost, $K_{p}$, in cases where batches of items perish; however, most of the models do not include the latter cost.

A more detailed discussion of the stateof-the-art results for each of these models is given in the article titled Mathematical
Models for Perishable Inventory Control in this encyclopedia.

\section{Discrete Review Systems}

Models with No Fixed Order Setup Cost and No Lead Time. The simplest model is one in which items' shelf-life equals the review period. Then, different periods are independent and the standard newsvendor solution is optimal in each period. The second simplest model, in which items' shelf-life equals two review periods, is already much more interesting. This model was pioneered in Ref. 6, where it was shown that the optimal control policy is state dependent.

That the optimal control policy is state dependent, is somewhat surprising, because the optimality of the base stock control policy is easily established in discrete review models for nonperishables without order setup cost. Moreover, this result is also important because it implies that for models in this category, the simple base stock-level control is not optimal, in sharp contrast to the optimality of this control for nonperishables.

Thus, for perishable items, the optimal inventory control requires solving a dynamic programming problem. The difficulty in finding and implementing the optimal state-dependent controls motivated researchers to search for effective heuristics. For example, Broadheim et al. [7] suggested a heuristic that is based only on the age of the newest item in the system and the simulation-based study in Ref. 8 considered several heuristics including one that keeps the total number of items in the system fixed, ignoring their ages.

Models with Fixed Ordering Cost but no Lead Time. The research on this category was pioneered in Ref. 9, which highlights the complexity of the optimal control structure for these models and suggests heuristic for control of the system based on the $(S, s)$ model.

A nice observation for this model of the Poisson demand with backlogs case is that the optimal reorder level is always at or below -1 . This result, which was established in Ref. 10, echoes the one in Ref. 11 for the continuous review system. The proof follows 
because - with no lead time-higher reorder points would increase holding cost without reducing the backlog. (This result also holds in the case where customer's arrival process is Poisson and each customer requests some integer quantity of the product.)

Models with Lead Time. The inclusion of positive lead time leads to much more complicated models, as the number of states required for the dynamic program increases. With lead time, one needs to also keep track of the age of ordered items. This model is considered in Ref. 12, where methods to solve it are suggested. Recently, Berk [13] analyzed the $(Q, r)$ periodic review inventory model for perishables with lead time by using the effective shelf-life distribution and assuming lost sales.

Discussion. The assumption of zero lead time in the case of a discrete review system is often justified if the lead time is shorter than the review period. Therefore, the analysis of such models (with periodic review and no lead time) is valuable. The zero lead time assumption is not as meaningful for continuous review models or when the lead time is long and makes reviewing the items at such intervals too expensive.

From an analysis point of view, treating perishable items requires consideration of the age distribution of the on-hand inventory in both ordering and dispatching of perishable items. Dispatching is also important because, typically (and in contrast to Example 1), the value of older items in stock is lower than that of the newer ones. The challenges in both ordering and dispatching of perishables causes the traditional optimal policies for nonperishable items to be suboptimal for perishables.

It turns out that the optimal control policies for perishable items are fairly hard to characterize. This difficulty encouraged researchers to develop effective heuristics for the periodic control of perishable items in the absence of lead time. However, no such heuristics were developed for models with long lead time (Williams and Patuwo [12] give an exact analysis of several relevant cases and suggest guidelines for developing such heuristics).

\section{Continuous Review Systems}

Models without Order Setup Cost or Lead Times. This category was motivated to model blood banks. It was originated by Graves [14], who assumed that the items are continuously produced, perish after a deterministic time, and that demand follows a compound Poisson process with either a single unit or an exponential demand at each arrival. A large body of work within this category was done by Perry et al. in Ref. 15 and references therein; thus Karasesman et al. [2] named it the Perry model.

An interesting observation that is used in the analysis of the Perry model is that its performance can often be characterized based on knowledge of the virtual death process, that is, the time until the next perishability [16].

The main assumption made in many of the models within this category is that good items arrive one by one independently of the decision making. Thus, in contrast to standard inventory models, the controller does not decide on when and how much to order. Still, the controller might affect the arrival rate of good items by advertising, for example.

Because of this lack of control on the input, most work on the Perry model focused on performance analysis rather than on finding optimal controls.

Models without Order Setup Cost but with Positive Lead Time. The first work on continuous review models without setup cost but with lead time is given in Ref. 17 where, as in many subsequent works, the performance of an $(S-1, S)$ control policy is investigated. However the optimality of this control policy is not established, even when time to perishability and interarrival times are exponential. In fact, in view of the fact that base stock is not the optimal policy in the corresponding discrete review model, even in the absence of lead time, this control policy is probably not optimal for the continuous review models either. While some study of problems with fixed lead time and time to perishability has been pursued [18], the theoretical results about these applicable models are far from being complete. 
Models with Order Setup Cost. Models with order setup cost can be portioned into ones with a fixed or variable order size, corresponding to the $(Q, R)$ or the $(s, S)$ standard inventory models. Both types of models can be analyzed as cost minimization problems, possibly subject to some service level constraint.

For fixed batch size problems, two policies were considered: the standard $(Q, R)$ and the $(Q, R, T)$ policy, where orders of a batch are triggered by either the time passed (since the inventory level was $Q$ ) being $T$, or by the inventory level falling below $R$ [19].

The vast majority of work allowing for variable order size assumes no lead time, as in Ref. 11. In Ref. 11, it is established that with Poisson demand the $(S, s)$ is the optimal policy when shortages are either backlogged or result in lost sales, and also that $s<-1$ or $s=0$ for these models, respectively. (The insight behind the proof on the optimal reorder point is similar to the one for the corresponding discrete review model discussed in the section titled "Models with Fixed Ordering Cost but no Lead Time.")

Recently, Gurlur and Ozkaya [20] considered a zero lead time with backlog $(s, S)$ policy for perishables. They used sums and integrations of relevant distribution functions to express the expected cost rate function for their model and developed a heuristic for the positive lead time case.

Discussion. To summarize, stochastic analysis is the main tool used in the investigation of continuous review models for perishable items. This analysis is not trivial and the characterization of the optimal control policies is more complicated than in the periodic review models. Therefore, there is a need to find effective control policies for these models as well, especially in the presence of lead time.

There is still a place for developing efficient heuristics for the control of continuous review policies for perishables with lead time. Both the heuristic reported in Ref. 20 and the one for the no lead time case from Ref. 3 , which is also reproduced in the next section, seem good starting points for finding such heuristics.

\section{DEVELOPING A HEURISTIC CONTROL POLICY}

As discussed above, there is a need for developing effective control policies for both, continuous and discrete review models of perishable items. Below, we discuss such a heuristic based on Ref. 3. Consider a continuous review model with no lead time, where the customers arrival process is Poisson with rate $\lambda$ and each customer requests a continuous random quantity $D$ of items with a mean demand size of $E(D)$. The costs considered are the order setup cost, $K$, holding cost per item per time period, $h$, and cost of perishable items, $c_{p}$. Let us focus on an $(S, s)$ control policy and allow no backlog, which is legitimate due to the zero lead time assumption. Thus, $s=0$ is optimal and due to the Poisson arrival process, the times where the inventory level is raised to $S$ are renewal epochs (see Definition and Examples of Renewal Processes, properties of renewal processes)

In the standard $(S, 0)$ model without perishable items, the end of the inventory cycle, denoted by $T_{S}$ is caused only due to the arrival of a demand. Of course, $T_{S}$ is a random variable that depends on $S$ and on the demand arrival process. But when items are perishable, cycles can also end due to perishability, at time $L_{p}$, which is the random variable describing the time to perishability of a new batch. To emphasize that the model focuses on perishable items, we denote the time to end the cycle by $\tau=\min \left(T_{S}, L_{p}\right)$ and the corresponding control policy by $(S, \tau)$, as in Ref. 3.

Figure 1 shows a sample path of inventory over more than two inventory cycles. The first cycle ends due to the demand, as in a standard $(S, 0)$ model; then $\tau=T_{S 1} \leq L_{p 1}$. The second cycle ends due to perishability; then $\tau=L_{p 2}<T_{S 2}$.

Let $V(t)$ denote the inventory level at time $t \in[0, \tau)$ from the beginning of the cycle. Thus, $\mathbf{V}=\{V(t): t \geq 0\}$ is a regenerative process such that $V(0)=S$. We let $V(\tau)$ denote the inventory level at the end of the cycle and use $E$ as the expected value operator (both $E(V)$ and $E(V(\tau))$ are well defined because $\mathbf{V}$ is a regenerative process). Then, the long-run 
Sample path $(S, \tau)$ models.

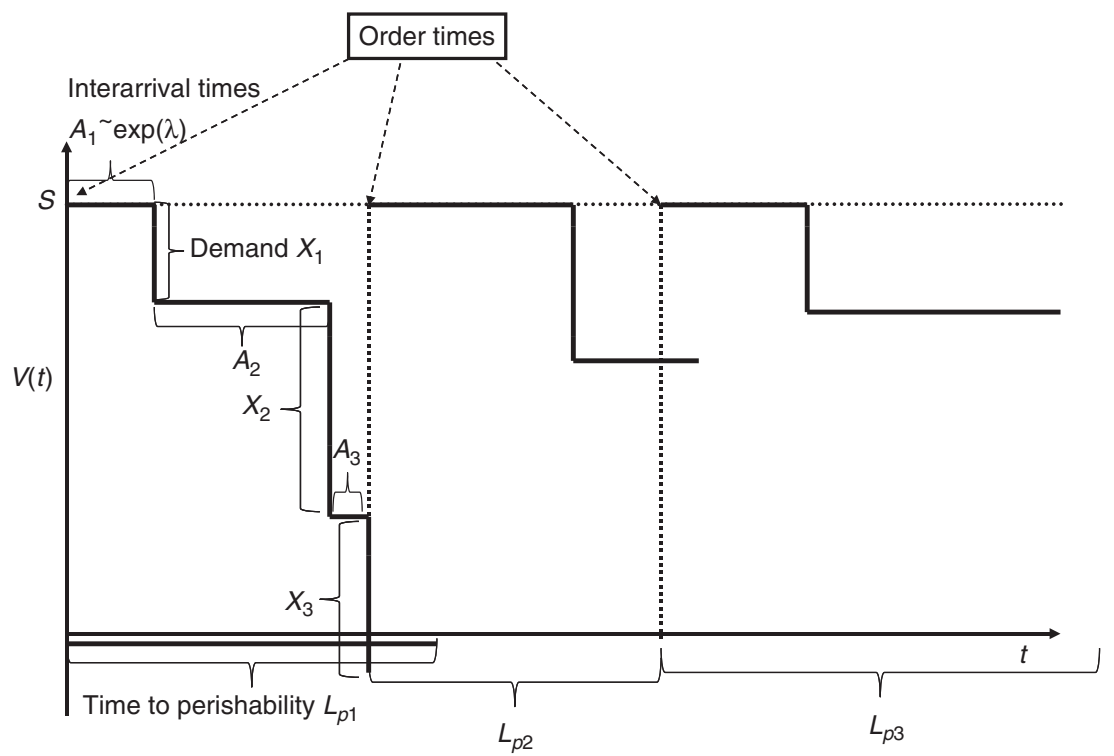

Figure 1. Sample path $(S, \tau)$ models.

average cost $C(S)$ is

$$
\begin{aligned}
C(S)= & \frac{K+c_{p} E\left(V(\tau) I\left\{\tau<T_{s}\right\}\right)}{E(\tau)} \\
& +h E(V)
\end{aligned}
$$

where $I\{\cdot\}$ is the indicator function with a value 1 , if the event $\{\cdot\}$ occurs and 0 otherwise.

To find a heuristic control policy, we replace the stochastic demand assumption with a deterministic demand with a rate $\lambda E(D)$. Then, starting at the reorder level $S$ for any time $t$ before the end of the cycle, we have

$$
V(t)=S-t \lambda E(D) \quad t \in[0, \tau) .
$$

Therefore, if items do not perish within a cycle its length is $T_{S}=S /(\lambda E(D))$. Letting $f_{L_{p}}(t)$ denote the pdf of $L_{p}$, the expected cycle length is given by

$$
\begin{aligned}
E(\tau)= & E\left(\min \left\{L_{p}, T_{S}\right\}\right) \\
= & \int_{0}^{S /(\lambda E(D))} t f_{L_{p}}(t) \mathrm{d} t \\
& +\frac{S}{\lambda E(D)} \operatorname{Pr}\left(L_{p}>\frac{S}{\lambda E(D)}\right)
\end{aligned}
$$

Since for every $t<\tau$ we have $V(t)=S-$ $t \lambda E(D)$ and, as in the EOQ model, the average inventory resulting from an inventory triangle of height $Y$ is $Y / 2$, we have

$$
\begin{aligned}
E(V)= & \int_{0}^{S /(\lambda E(D))}\left(S-t \lambda E(D)+\frac{t \lambda E(D)}{2}\right) \\
& f_{L_{p}}(t) \mathrm{d} t+\frac{S}{2} \operatorname{Pr}\left(L_{p}>\frac{S}{\lambda E(D)}\right)
\end{aligned}
$$

and

$$
\begin{aligned}
E\left(V(\tau) I\left\{\tau<T_{S}\right\}\right)= & \int_{0}^{S /(\lambda E(D))}(S-t \lambda E(D)) \\
& f_{L_{p}}(t) \mathrm{d} t .
\end{aligned}
$$

Remark. This heuristic assumes a continuous and deterministic demand process, thus new demand arrives immediately after an old batch perishes. In practice, when there is no lead time, ordering the next batch after items perish can be postponed until the next customer's arrival. That is, the order at the end of the second cycle in Fig. 1 can be postponed until the first arrival in the third cycle. Such 
postponement would increase $E(\tau)$ and can be incorporated into the heuristic by adding the expected time to an arrival to the expected cycle length whenever cycles end due to perishability. In contrast, when the demand size is continuous, it is likely that when the cycle ends due to demand there is some demand that should be satisfied from the next batch. Then when no backlog is allowed, a similar postponement is not feasible for cycles that end due to demand. In any case, postponing orders when cycles end due to perishability might not be beneficial when lead time is positive. Thus, in the example given below, we ignore such postponement and use $E(\tau)$ as given in Equation (2).

Example 3. The heuristic when $L_{p} \sim$ $\exp (\xi)$ : Assuming $L_{p} \sim \exp (\xi)$ using Equations (2)-(4), we get the approximated control problem:

$$
\begin{aligned}
& \min _{S} C^{h}(S)=\left.\frac{K+\pi\left(S-\frac{\lambda E(D)\left(1-e^{-\xi \frac{S}{\lambda E(D)}}\right)}{\xi}\right)}{\frac{1-e^{-\xi \frac{S}{\lambda E(D)}}}{\xi}}\right) \\
&+h\left(S-\frac{\lambda E(D)\left(1-e^{-\xi \frac{S}{\lambda E(D)}}\right)}{2 \xi}\right)
\end{aligned}
$$

while in general there is no closed form solution to problem (5), it is easily solved numerically.

This heuristic, of solving Equation (5) was investigated in Ref. 3, for cases in which the time to perishability is exponential or deterministic, arrivals follow a Poisson process, and customers' demand is either a unit or exponentially distributed. For these cases, Baron et al. [3] expressed the optimal order up to level, that is, the one that minimizes Equation (1) denoted by $S^{*}$, and compared it to the order up to the level based on solving Equation (5), $S^{H}$. For these cases, the cost error of the heuristic is relatively small (around 2\% on average). Thus, Baron et al. [3] suggested that this heuristic be used for cases where $S^{*}$ cannot be found.

\section{FUTURE RESEARCH DIRECTIONS}

Below, we discuss several research directions and explain their importance. Many of these extensions have attracted significant research for standard items, but in our opinion, they are not addressed well enough for perishable items.

The most important research direction for managing inventory of perishables is to develop effective heuristics for inventory control of such items. Some heuristic control policies were developed earlier, such as by Nahmias [21], who discusses heuristics for the deterministic shelf-life case. However, developing effective heuristics for additional settings is still required. Such settings should include both continuous and periodic review systems and focus on cases with positive lead times. A closely related research direction is to provide theoretical guarantees on the performance of different heuristics, similar to the established efficiency of power of two policies for standard inventory items [22].

The standard assumption in the literature on continuously reviewed perishable items is that the planning horizon is infinite. Investigating the inventory control of perishables for finite horizons in such settings also deserves more research attention.

Four other issues that have garnered a lot of attention for standard inventory items are their management (i) in multiechelon settings, (ii) when aiming to coordinate the supply chain, (iii) for multiple items, and (iv) in the presence of competition (see Business Process Outsourcing multiechelon, multiple items and Supply Chain Coordination). An important feature in investigating these issues is internal transshipment. Many models addressing nonperishable items ignore internal shipments, because the cost of internal transshipments might offset their benefit. However, because transshipments of perishable items may reduce the proportion of perished items, the value of internal transshipments for perishable items is higher 
than for nonperishables. Thus, allowing internal transshipments is preferable in models that consider multiple sites.

Initial steps at investigating the above issues for supply chains of perishable items were taken (Section 4 in Ref. 2). For example, for the supply of blood in multiechelon settings (Ref. 23 and references within), consider both, a rotation (or recycling) policy and a retention one. In a rotation system at the end of each period (e.g., day) the supplier gathers all unsold (and not yet perished) items from the retailers and then supply them at the beginning of the next period; in a retention system, supplied items are left with the retailers until they are demanded or perished. However, the literature addressing issues (i)-(iv) for perishables typically considered fairly restrictive settings. Therefore, there is room for addressing these issues and developing effective management policies for managing the flow and allocation of perishable items in supply chains.

Note that to properly address these issues, investigation of the strategic planning of supply-chain networks is required for perishable items. Appropriate models for supply-chain networks require as input, the inventory control policy used and their performances (see Supply Chain Coordination). For example, locating warehouses and distribution centers should consider the costs implied by different supply-chain configurations. However, in the absence of efficient methods to manage the inventory within the supply chain and to estimate the implied inventory-related costs, designing the network of the supply chain might be of limited value.

Another model that is well established for standard items is joint manufacturing, storing, and allocation of products for different customer types. This is also relevant for perishable items. An example is the choice made at a central depot that serves several different retailers with different importance. Then, an allocation of items may need to consider performance measures as seen by the different customer types. Such measures of service levels could be on-stock availability of "fresh enough" items, fill rates, and so on. In fact, inventory control of perishable items subject to service level constraints even for a single customer type has won little attention. Such constraints could be quite specific in defining appropriate service levels in relation to the residual life of items sold. Customers might not be satisfied with only a high on-shelf availability, but may also demand that available items are fresh enough. Moreover, in some cases different customer types prefer different levels of freshness that require different storage processes. For example, the shelf-life of tomatoes is longer if they are kept on the vine, refrigerated (that may also reduce their flavor), or turned into a tomato paste, tomato juice, or ketchup. Thus, it is likely that formulating problems with different customer types would be very application dependent. Still, in view of the importance of these applications, for example in the health-care industry, this is a topic deserving further research.

The above discussion of different service levels and storage requirements for perishable items raises another question; namely, the effective analysis of the logistics required for the delivery of perishable items. Logistics planning for perishable items involves capacity, storage, production, and transportation decisions as well as their effect on items' shelf-life. For example, some fruits are delivered to North America in ships, with part of their ripening occurring during this long and often uncertain transportation window. Once ripened, such fruits are often stored at cold temperatures to increase their shelflife. Thus, managing the logistics process required to bring valuable items to final customers is not simple, and deserves further attention. Again, such research is likely to be application dependent.

An important issue that is unique for perishable items is the dispatching policy which may be complex even for a single customer type. In addition to the complexity highlighted by Example 1, the dispatching policy can also affect the demand. For example, consumers of food items such as milk, often check the "best before" date of items on the shelf. Thus, demand for a specific item depends not only on its availability on the shelf and its age, but also on the age and shelf availability of items of different ages. That is, there 
are substitutability effects among items that differ by age. In addition, in this setting, the controller can only affect rather than dictate the dispatching policy. This is a mirror problem to the models in the section titled "Models Without Order Setup Cost or Lead Times," where the controller can only affect rather than dictate the arrival of new items. Again, initial works on this subject exist (Section 5.2 of Ref. 2), but there is still plenty of opportunity for research in more realistic settings.

As the last but not the least research direction, we list the following simplifying assumptions that are often made in works on perishable items. Most of the references above assume that (i) demand is known (in case of stochastic demand its distribution is known), (ii) there is no seasonality, (iii) demand is independent of pricing, shelf space allocation, and inventory level, (iv) order yield is perfect, and (v) there is no substitutability of items (both within items of different ages and among different products). For nonperishable items, all of these assumptions have been relaxed to some extent. However, the vast majority of papers on managing inventory of perishables make these assumptions. (Several initial attempts at relaxing some of these assumptions are referenced in Section 5 of Ref. 2.)

\section{REFERENCES}

1. Grocery manufacturers of America. 2006 Unsaleables benchmark report. 2006.

2. Karaesmen I, Scheller-Wolf A, Deniz B. Managing Perishable and Aging Inventories: Review and Future Research Directions. Kempf K, Keskinocak A, Uzsoy P, editors. Handbook of Production Planning. Kluwer Academic Publishers; 2009. To appear.

3. Baron O, Berman O, Perry D. Stochastic $(\tau, \mathrm{S})$ models for managing inventory of perishable items. Working paper. Rotman School of Management, University of Toronto; 2008.

4. Rajan A, Steniberg R. Dynamic pricing and ordering decisions by a monopolist. Manag Sci 1992;38:240-262.

5. Brown GW, Lu JY, Wolfson RJ. Dynamic modeling of inventories subject to obsolescence. Manag Sci 1964;11(1):51-63.

6. Nahmias S, Pierskalla W. Optimal ordering policies for a product that perishes in two periods subject to stochastic demand. Nav Res Logist Q 1973;20:207-229.

7. Broadheim E, Derman C, Prastacos GP. On the evaluation of a class of inventory policies for perishable products such as blood. Manag Sci 1975;22:1320-1325.

8. Nahmias S. A comparison of alternative approximations for ordering perishable inventory. INFOR 1975;13:175-184.

9. Nahmias S. The fixed charged perishable inventory problem. Oper Res 1978;26: 441-481.

10. Lian Z, Liu L. A discrete-time model for perishable inventory systems. Ann Oper Res 1999;87:103-116.

11. Weiss H. Optimal ordering policies for continuous review perishable inventory models. Oper Res 1980;28:365-374.

12. Williams CL, Patuwo BE. A perishable inventory model with positive order lead times. Euro J Oper Res 1999;116:352-373.

13. Berk E, Gurler U. Analysis of the $(Q$, r) inventory model for perishables with positive lead times and lost sales. Oper Res 2008;56(5):1238-1246.

14. Graves S. The application of queuing theory to continuous perishable inventory system. Manag Sci 1982;28:400-406.

15. Nahmias S, Perry D, Stadje W. Actuarial valuation of perishable inventory systems. Probab Eng Infor Sci 2004;18:219-232.

16. Kaspi H, Perry D. Inventory system of perishable commodities. Adv Appl Probab 1983;15:674-685.

17. Pal M. The $(s-1, S)$ inventory model for deteriorating items with exponential lead time. Calcutta Stat Assoc Bull 1989;38:83-91.

18. Perry D, Posner M. An $(S-1 ; S)$ inventory system with fixed shelf-life and constant lead times. Oper Res 1998;46:65-71.

19. Tekin E, Gurler U, Berk E. Age based vs stocklevel control policies for a perishable inventory system. Eur J Oper Res 2001;134:309-329.

20. Gurlur U, Ozkaya BY. Analysis of $(s, S)$ policy for perishables with a random shelf-life. IIE Trans 2008;410:759-781.

21. Nahmias S. Perishable inventory theory: a review. Oper Res 1982;30(4):680-708.

22 . Roundy R. A 98\% effective integer ratio lotsizing rule for a one warehouse multi-retailer, system. Manag Sci 1985;31:1416-1430.

23. Prastacos GP. Allocation of a perishable product inventory. Oper Res 1981;29:95-107 